\title{
ANALYSIS OF DELAY-TOLERANT ROUTING PROTOCOLS USING THE IMPACT OF MOBILITY MODELS
}

\author{
MD. SHARIF HOSSEN*AND MUHAMMAD SAJJADUR RAHIM ${ }^{\dagger}$
}

\begin{abstract}
Intermittently connected mobile networks are sparsely connected wireless ad-hoc networks where there is no endto-end path from a source device to a destination. Generally, these paths do not exist. Hence, these devices use intermittent paths using the concept of store-and-forward mechanism to successfully accomplish the communication. These networks are featured by long delay, dissimilar data rates, and larger error rates. Hence, we look into the analysis of several delay-tolerant routing protocols, e.g., epidemic, spray-and-wait, prophet, maxprop, rapid, and spray-and-focus using opportunistic network environment simulator. At first, the investigations of the above considered routing protocols are done across three mobility models namely random direction, random walk, and shortest path map based (SPMB) movement model for node impact only. Then, we evaluate these routing protocols against the impacts of message copy, buffer, and time-to-live using SPMB movement model considering the results of node impact. We use three metrics, and the results show that spray-and-focus yields good performance for showing higher delivery, lower latency, and lower overhead among all routing techniques, while epidemic is poor.
\end{abstract}

Key words: Delay-tolerant network; ad hoc networks; communication; routing; replication; simulation

AMS subject classifications. $68 \mathrm{M} 12,68 \mathrm{U} 20$

1. Introduction. With the increase of portable devices (e.g. smartphones, laptops), a class of ad-hoc networks have become popular nowadays $[1,2,3,4,5,6,7,8]$ which are known as delay-tolerant networks (DTNs) [9]. DTNs [10] are also called intermittently connected (IC) [11]. These are wireless mobile adhoc networks where devices can not build an end-to-end route. Examples of such networks are satellite communication [12], wildlife tracking, [13], military, and vehicular [14, 15].

However, these networks use a technique named store-carry-and-forward to successfully communicate among mobile devices $[16,17,18,19,20,21]$ by passing the information to intermediate devices, where messages are stored in respective buffer and are forwarded to other relays in the network. Message copy passing can be in two ways, firstly only using a single copy called forwarding based and secondly using two or more copies called replication based [22, 23, 24].

Only replication based routing techniques mentioned in Sec. 2 are used in this research. Three mobility models are used to analyse their impact on the performance of routing protocols in the considered IC mobile network scenario. The performances are analysed for changing message copies, node impact, TTL, and buffer on the impact of mobility models. Using ONE simulator we see that spray-and-focus shows good performance compared to all and epidemic poor.

Remaining part of this research is written as follows. In Sec. 2, we discuss the classification of routing strategies. Section 3 involves different parameter setting with simulator description. Different mobility models are summarized in Sec. 4. Then, the explanation of different arguments is included in Sec. 5. Finally, the summary with future endeavors is discussed in Sec. 6.

2. Routing Protocols. This section summarizes the general classification and description of routing techniques.

2.1. Classification of Routing. Generally, two categories of routing protocols are single-copy and multicopy. In the first case, only one message transmission through the nodes is possible [11, 30]. In the second case, two or more message transmissions at a time are possible in the network [25]. The first case exhibits lower message transmission with the higher delay due to network partition [26, 27]. While, the second case can provide better delivery due to the message replication [28, 29].

\footnotetext{
*Lecturer, Department of Information \& Communication Technology, Comilla University, Comilla-3506, Bangladesh (sharif5613@gmail.com).

${ }^{\dagger}$ Associate Professor, Department of Information \& Communication Engineering, University of Rajshahi, Rajshahi-6205, Bangladesh, (sajid_ice@ru.ac.bd).
} 
2.2. Forwarding Based. Here, we discuss forwarding based routing techniques. As an example, we consider the source device as $\mathrm{X}$, the destination as $\mathrm{Z}$, and intermediate devices can be any of the following symbols: Y, P, Q, R, S.

First Contact. Using this routing, a device can send a copy to the nearest first device found. As an example, the source device X can forward a single copy to the first available device (say Y). After successfully sending a message to $\mathrm{Y}$, the device $\mathrm{X}$ does not store any copy. $\mathrm{Y}$ can also forward a message similar to $\mathrm{X}$, and this process continues until found $\mathrm{Z}[26]$.

Direct Delivery. Using this routing, a device can send a message directly to a device without sharing its copy to anyone. That is, no intermediate nodes are used here. As an example, source device X directly sends the message to $\mathrm{Z}$ without taking any help of intermediate devices: Y, P, Q, etc. So, this technique [31] faces high delay.

2.3. Replication Based. Here, replication based routing techniques are summarized. we consider the source device as $\mathrm{X}$, the destination device as $\mathrm{Z}$ and the intermediate devices as $\mathrm{Y}, \mathrm{P}, \mathrm{Q}, \mathrm{R}, \mathrm{S}$, etc.

Epidemic. This routing is the first among the replication based concepts where total replicas are very high. Hence is named as flooding. As an example, $\mathrm{X}$ can generate and forward several message copies to the intermediate devices, say, P, Q, R, S, which can store a message copy if they do not have that copy. These devices then replicate and continue this process until found $\mathrm{Z}$ [32, 33]. This routing exhibits much more overhead for its replication process [22].

Prophet. Using this routing, if $\mathrm{X}$ wishes to forward a message copy to its intermediate devices, say, $\mathrm{P}, \mathrm{Q}$, then depending on the past record of delivery, $\mathrm{X}$ forwards the message to either $\mathrm{P}$ or $\mathrm{Q}$, i.e., if the probability of meeting $\mathrm{Z}$ from $\mathrm{P}$ is greater than the probability of meeting $\mathrm{Z}$ from $\mathrm{Q}$, then $\mathrm{X}$ forwards the message to $\mathrm{P}$, and $\mathrm{P}$ then easily contact to $\mathrm{Z}[33]$.

Prophet version-2. Prophet suffers from the delivery problem if the transitivity among nodes is not zero. Prophet version 2 solves this issue by calculating the maximum probability among two frequently encountered nodes having transitivity[34].

Rapid. Using this routing, devices can use one of the three optimization metrics, i.e., reduction of average delay, missed deadline, and maximum delay. Hence, the devices can forward a message to a relay calculating the utility value, which is related to the bandwidth, and the buffer capacity in the network [35].

Maxprop. Using this routing, a device forwards a message copy to an intermediate device having lower hop counts using Dijkstra shortest path algorithm [36].

Spray-and-wait (SNW). This routing has two variations: vanilla, and binary. In the first variation, $\mathrm{X}$ can send $\mathrm{N}$ copies to the first $\mathrm{N}$ devices where all devices including $\mathrm{X}$ are in the waiting phase to contact $\mathrm{Z}$. While, in the second variation, $\mathrm{X}$ having $\mathrm{N}$ copies sends $\mathrm{N} / 2$ copies to $\mathrm{P}, \mathrm{Q}, \mathrm{R}$, and $\mathrm{S}$ where only one device, say, $\mathrm{P}$ receives $\mathrm{N} / 2$ copies. If a device has a copy, then it can not store another copy. $\mathrm{P}$ again sends $\mathrm{N} / 4$ copies to the next first devices available and continues it until having a single copy (say $\mathrm{S}$ carrying it). This indicates that $\mathrm{S}$ is in the waiting phase and directly contacts Z [37]. In this work, binary version (B-SNW) has been used.

Spray-and-focus (SNF). A device having only a single copy is in the waiting phase as the description of SNW, but SNF routing [38] forwards this single copy to another relay instead of destination which is the focus phase having a criteria as following: $\mathrm{P}$ can forward a single copy instead of $\mathrm{Z}$ to $\mathrm{Q}$ if the utility of $\mathrm{Q}$ to meet $\mathrm{Z}$ is greater than the utility of $\mathrm{P}$ to contact $\mathrm{Z}$ plus a fixed value.

3. ONE Simulator with Simulation Setting. We discuss here ONE simulator and the parameters used in the simulation.

3.1. ONE Simulator. ONE is a java unified mapping platform to trace the visual contact of mobile nodes, message passing, event generation of real scenario which is packaged as a single java project in [31, 39, 40]. A simple simulation scenario is shown in Fig. 3.1. 


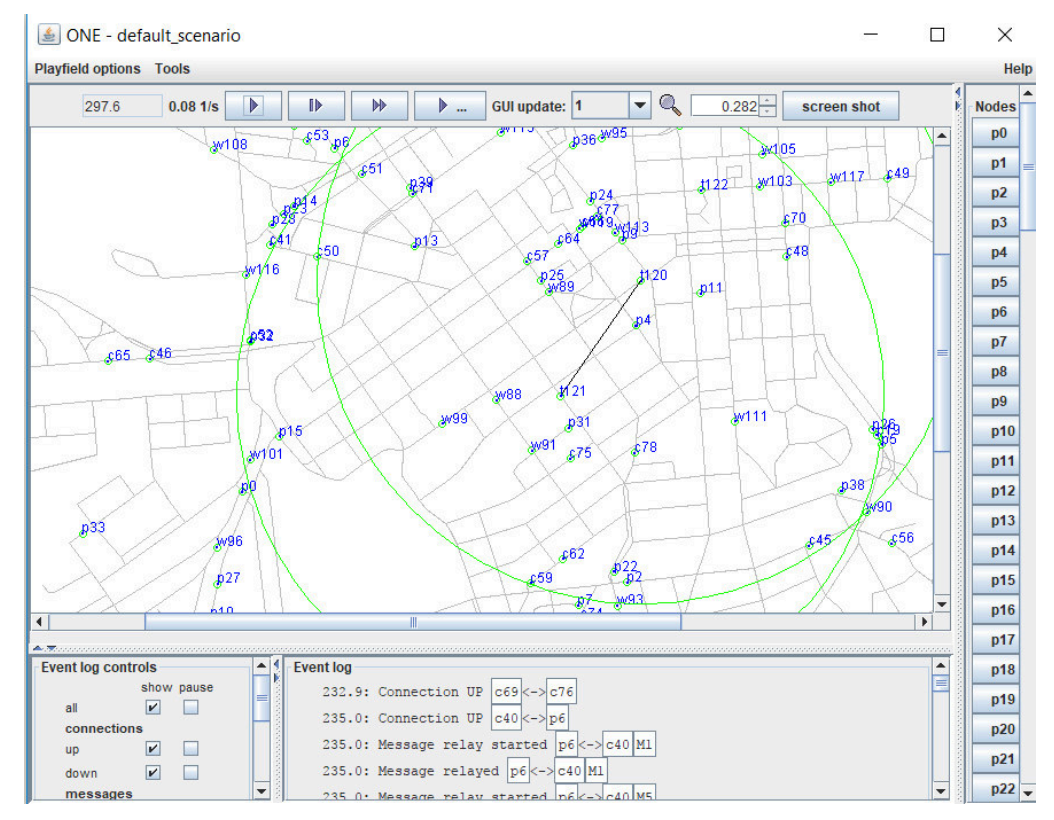

FIG. 3.1. Simulation scenario.

3.2. Simulation Setting. During the evaluation, we have used a laptop: HP Pavilion, processor: Intel(R) Core(TM) i7-6500U CPU @ 2.50 GHz, installed memory (RAM): 8.00 GB, and 64-bit OS. In the simulation, we have used various parameters which are summarized as follows. We have used 24 hours as the simulation time, Bluetooth as an interface with transmit velocity $250 \mathrm{kbps}$ and range $10 \mathrm{~m}$. Message size has been in the range $500 \mathrm{~KB}$ to $1 \mathrm{MB}$. We have considered simulation area size as $4500 \mathrm{~m}$ x $3400 \mathrm{~m}$ (width $\mathrm{x}$ height). The fixed values for message copies, number of hosts, buffer, and time-to-live are 2 (msg/min), 100, 5 MB, 300 (minutes) respectively. When one parameter is changed, other values are kept at fixed. Hence, we have changed the message copies as 2, 3, 4, 5, 6, and 10 ( $\mathrm{msg} / \mathrm{min})$. Node's buffer size has been changed as the values of 5 , 10, 15, 20, 25 (MB). We have changed time-to-live as 50, 100, 150, 200, 250, and 300 (minutes). In this case, during the change of TTL $(50,100,150,200,250$, and 300 minutes), the fixed values for message copies, number of hosts, and buffer size are $2(\mathrm{msg} / \mathrm{min}), 100$, and $5 \mathrm{MB}$.

4. Mobility Models. We have considered three mobility models which are summarized below.

4.1. Random Walk (RW). Using this mobility [41, 42, 43], devices randomly move from one location to another until found the destination with a fixed speed within a predefined range.

4.2. Random Direction (RD). There are three categories of this mobility $[44,45,46]$. In the first case (we use here) [44], a device with a predefined speed starts to move in a particular direction randomly until hits the boundary of the simulation area. After touching the boundary wall, it takes a pause and moves to another direction to meet the destination.

4.3. Shortest Path Map Based (SPMB) Movement. This mobility uses the Dijkstra algorithm to find out the shortest path between two devices [47].

5. Results and Discussion. Firstly, we see the analysis of three mobility models discussed in Sec. 4 using node impact. Then, taking decisions from the results we investigate the DTN routing protocols' performance for changing various impact of parameters.

5.1. Mobility Based Node Impact on DTN Routing. Here, we analyze the node impact on the mobility models. 
5.1.1. Delivery. Both RW and RD follow the random nature but different strategy to trace a path from one to another position, whereas SPMB movement model follows Dijkstra strategy to determine the minimum distance of reaching a node from other. Hence, we see from Fig. 5.1 that all routing techniques provide higher delivery using SPMB mobility while lower delivery for RD in the considered scenario.

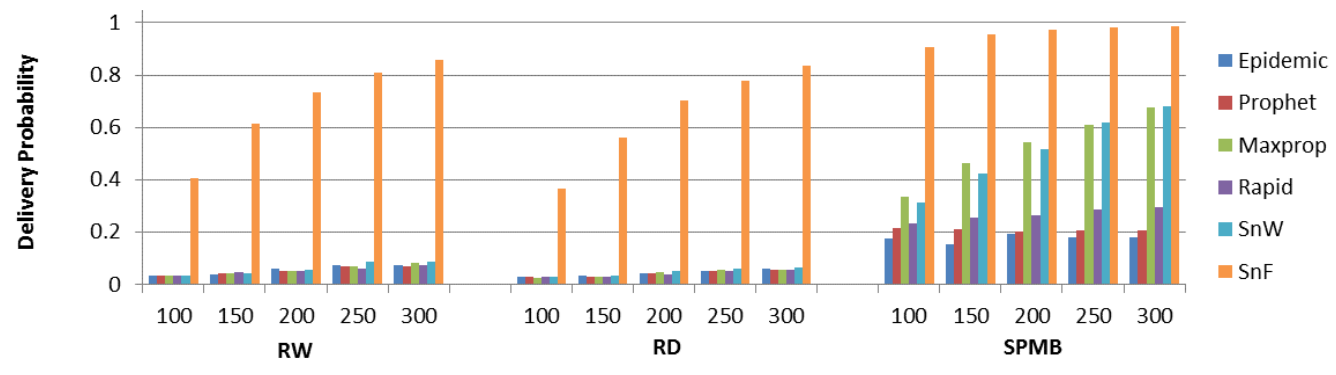

FIG. 5.1. Delivery probability with varying number of nodes.

5.1.2. Latency. From Fig. 5.2, we see that with the increase of devices in the network, latency increases for three cases i.e., RW, RD, and SPMB. However, we see lower latency using SPMB mobility while higher latency in other two mobility models. Therefore, we can conclude that SPMB will show the desired performance in our scenario.

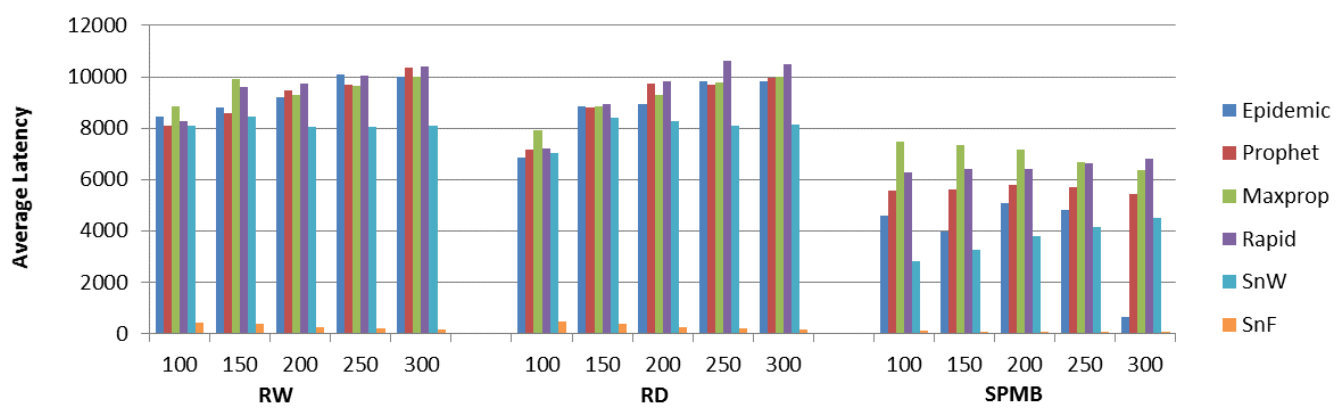

FIG. 5.2. Average latency with varying number of nodes.

5.1.3. Overhead. Although the overhead is higher for all routing strategies except for SNF and SNW using SPMB mobility while lower in other two mobility models, we see that SNF and SNW have lower overhead in SPMB mobility rather than using RW and RD as shown in Fig. 5.3.

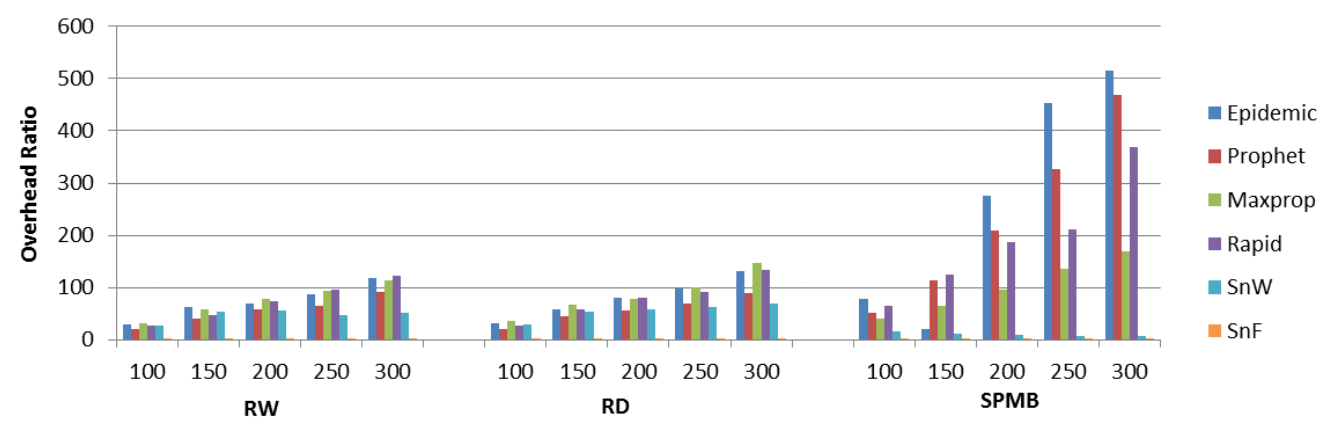

FIG. 5.3. Overhead ratio with varying number of nodes. 
Therefore, considering our scenario it is apparent that SPMB mobility is preferred instead of using RW and RD due to ensure higher delivery, lower latency and lower overhead (in the case of B-SNW and SNF).

5.2. SPMB Analysis on DTN Routing. From the investigation of Sec. 5.1, it is evident that for our scenario routing techniques show better performance using SPMB mobility instead of using RW and RD. We do the analysis of routing techniques discussed in Sec. 2.3 using SPMB mobility for changing certain network parameters with respect to three metrics.

5.2.1. Delivery with Message Copies and Node Density. In replication based routing, major problem is to control the limit of replicating of messages. More techniques have been proposed where SNF can control the maximum replication while Epidemic can forward the copies randomly with the nature of flooding. So, in both cases (shown in Figs. 5.4(a), and (b)) i.e., for changing message copies and mobile devices, we see that SNF routing exhibits much delivery while Epidemic the less. Since rapid routing needs more resources for the heavy density of devices, we can not include this in Fig. 5.4(b) due to the complexity of calculation.

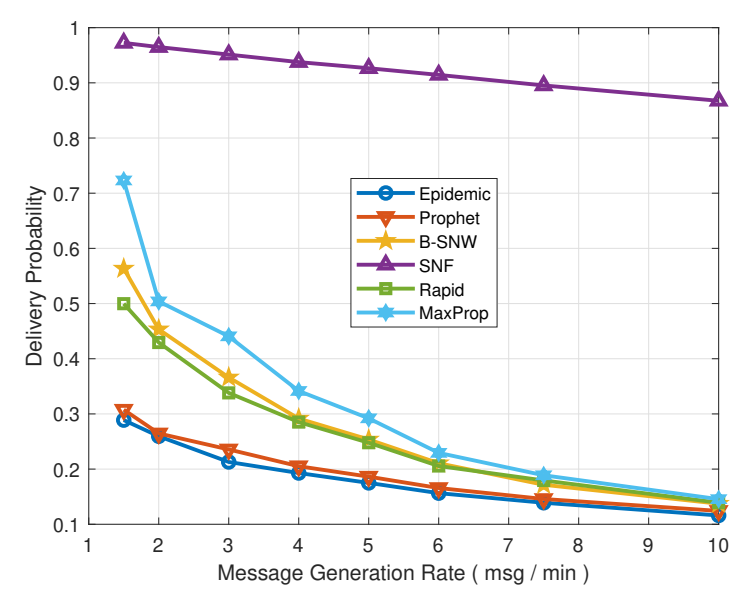

(a) Delivery probability with changing message copies.

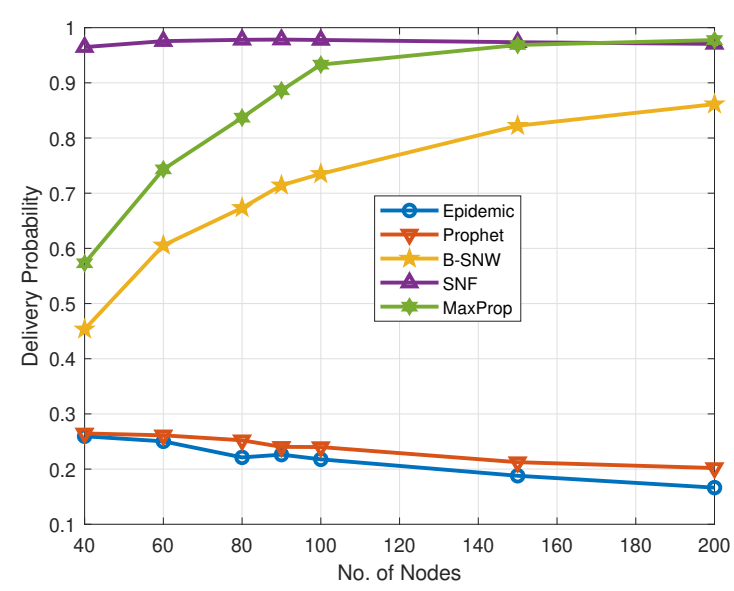

(b) Delivery probablity with changing node density.

FIG. 5.4. Delivery probability with changing message copies and node density.

5.2.2. Delivery with Buffer and TTL. We know if we have more space, then we can store more soft materials. The same thing here is that with the increase of buffer, devices can store more copies that's why delivery increases. While TTL is a lifetime of a message copy to exist in the network and so increases delivery. In both cases shown in Figs. 5.5(a) and 5.5(b), we see better performance for SNF while lower for Epidemic and Prophet.

Therefore, from the investigation of Sec. 5.2.1 and 5.2.2, it is clear that SNF routing shows better delivery and Epidemic the lower compared to other routing protocols mentioned in Sec. 2.3.

5.2.3. Latency with Message Copies and Node Density. We see that with the increase of message copies and mobile devices, latency decreases for all routing techniques except for B-SNW which increases latency for increasing devices. In both cases, we see lower delay for SNF and higher for Maxprop as shown in Figs. 5.6 (a) and 5.6 (b). For the same reason discussed in 5.2.1, we can not include Rapid routing (Fig. 5.6 (b)).

5.2.4. Latency with Buffer and TTL. As shown in Figs. 5.7 (a) and 5.7 (b), we see that latency increases with the increase of buffer and TTL for all routing techniques except Maxprop (greater than the latency in SNF). For varying buffer, Rapid and Epidemic shows very high latency because Epidemic does not follow any strategy for the limit of copies of messages while Rapid is resource thirsty which causes more delay of transferring copies from one to another. While for changing TTL, Maxprop provides very high growing latency. Latency for SNF is constant and lower for changing TTL. In both cases, we see lower delay for SNF. 


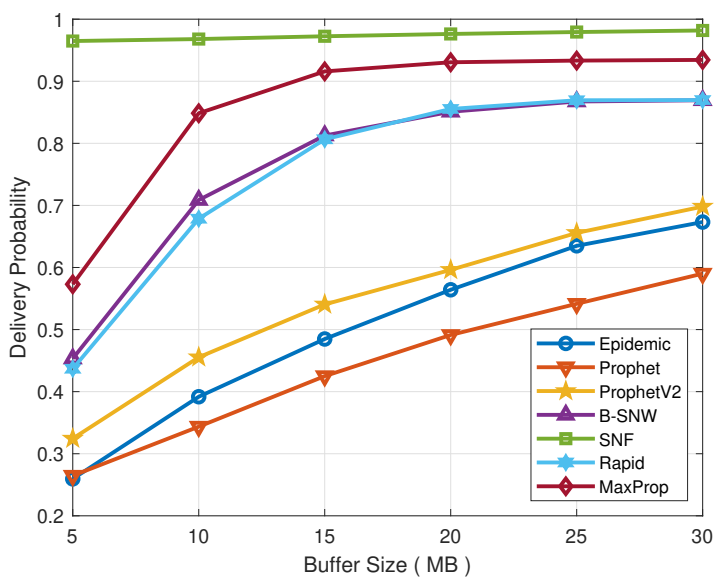

(a) Delivery probability with varying buffer size.

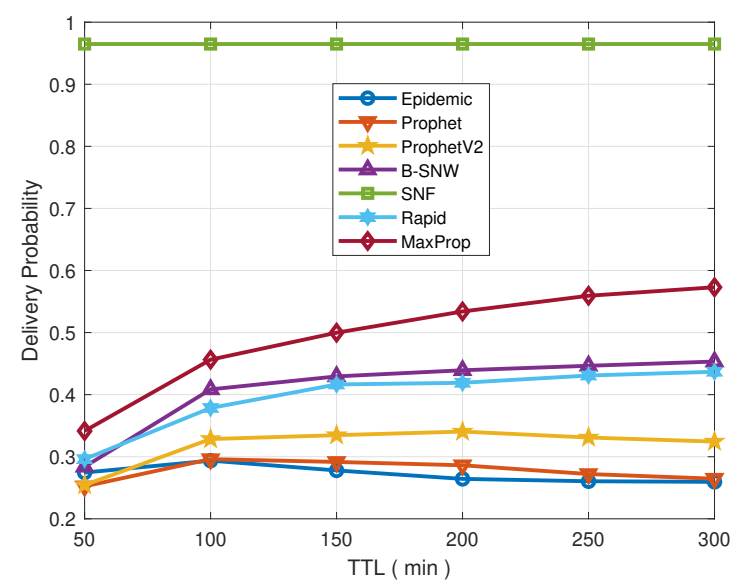

(b) Delivery probability with varying TTL.

FIG. 5.5. Delivery probability with varying buffer and TTL.

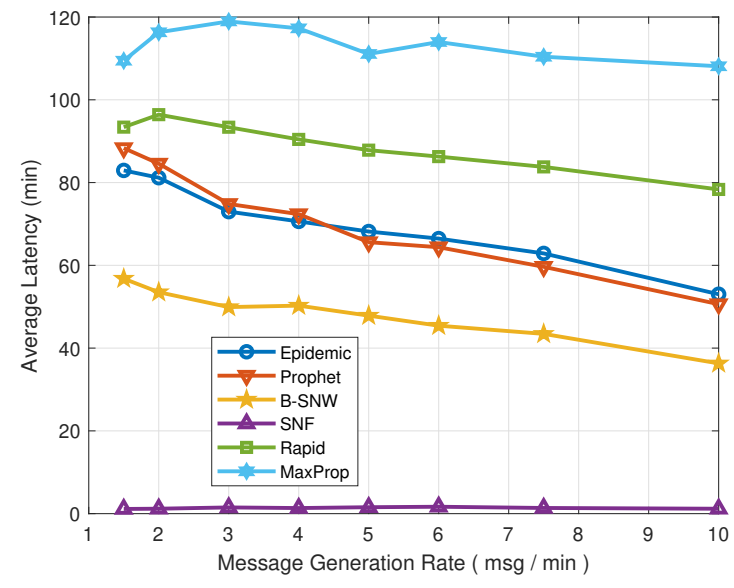

(a) Average latency with varying message copies.

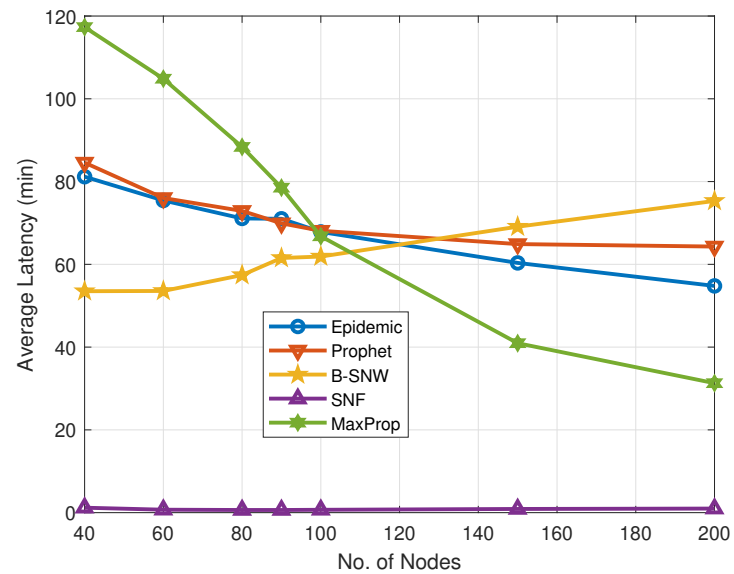

(b) Average latency with varying node density.

FIG. 5.6. Average latency with varying message copies and node density.

Therefore, from the investigation of Sec. 5.2.3 and 5.2.4, it is clear that SNF shows lower delay compared to all routing techniques mentioned in Sec. 2.3 while Maxprop the higher for changing message copies, node density, and TTL. For changing buffer size, Epidemic and Rapid shows high latency.

5.2.5. Overhead with Message Copies and Node Density. As the above discussion we know that Epidemic uses the concept of widespread outbreak of something, i.e. message copies which causes the chance of congested network while SNF follow the most technical way to limit the replication of copies. Hence, for this reason, we see higher overhead for Epidemic and lower for SNF in both cases i.e., for changing message copies and number of devices as shown in Figs. 5.8 (a) and 5.8 (b). Rapid has not been simulated for the cause discussed above.

5.2.6. Overhead with Buffer and TTL. From the discussion of Epidemic and SNF in 5.2 .5 we see greater overhead for Epidemic and lower for SNF in both cases i.e. for changing buffer and TTL as in Figs. 5.9 (a) and 5.9 (b). In Fig. 5.9 (a), we see Prophet has higher overhead than Epidemic since it stores the record of encountering a node in buffer to forward a copy to the next node. 


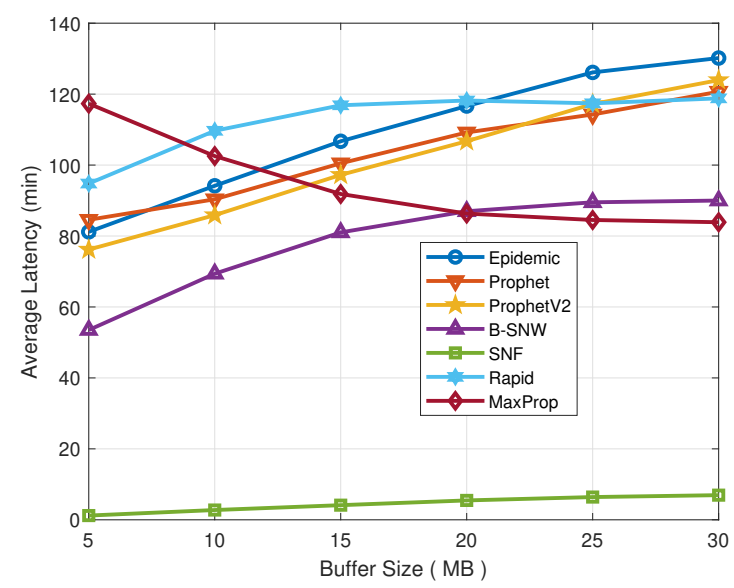

(a) Average latency with changing buffer size.

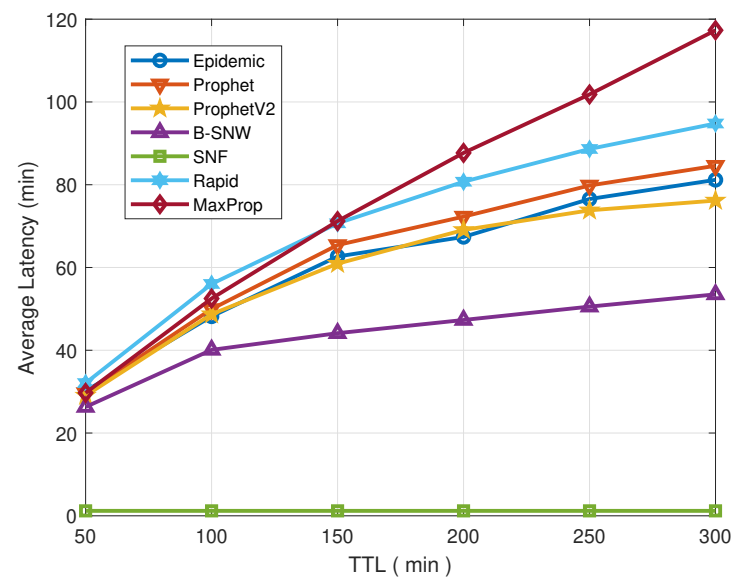

(b) Average latency with changing TTL.

FIG. 5.7. Average latency with changing buffer and TTL.

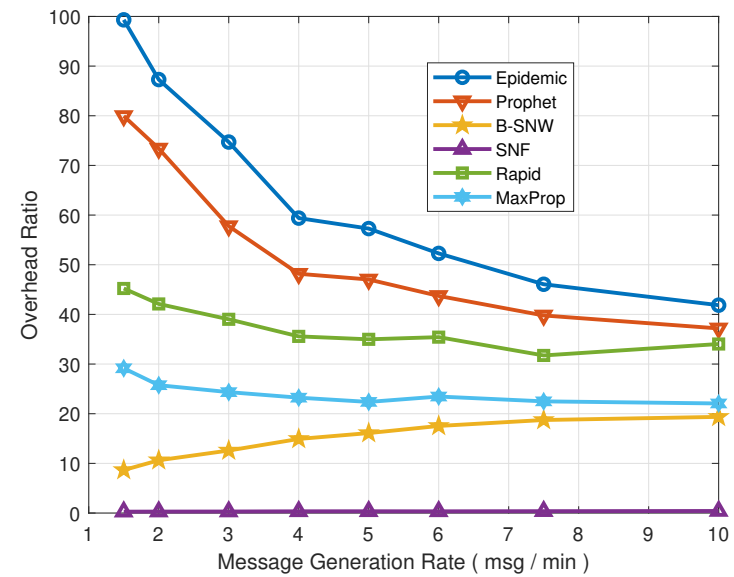

(a) Overhead ratio with changing message copies.

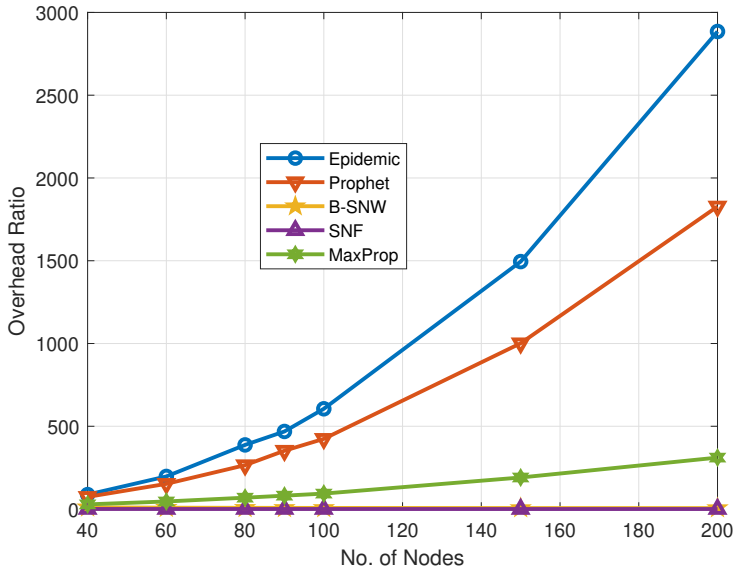

(b) Overhead ratio with varying mobile nodes.

FIG. 5.8. Overhead ratio with changing message copies and density of devices.

Therefore, from the investigation of Sec. 5.2.5 and 5.2.6, it is clear that SNF routing shows lower overhead ratio and Epidemic the higher compared to other routing protocols mentioned in Sec. 2.3 except for the case of varying buffer where Prophet shows higher overhead.

6. Conclusion and Future Works. In delay tolerant network, devices need to be intermittent as they use the store-and-forward model to deliver a packet successfully to the desired device. This intermittent ad hoc network is featured by topology partitions, long delays, etc. In this research paper, at first we see the impact of three mobility models namely random walk, random direction, and shortest path map based movement mobility only for node density. Then, considering the impact of node density, we evaluate the performance of several DTN routing protocols namely Epidemic, Prophet, Prophetv2, Maxprop, Rapid, Binary spray-and-wait, and Sprayand-focus in an intermittently connected mobile network scenario against changing message copies, density of nodes, buffer and time-to-live using ONE simulator in terms of three performance measurements namely delay, delivery, and overhead. The investigated results demonstrate that spray-and-focus routing exhibits the best performance while epidemic the poor under the consideration of all the metrics.

In near future, we would like to extend this work by using opportunistic mobility models to evaluate the 


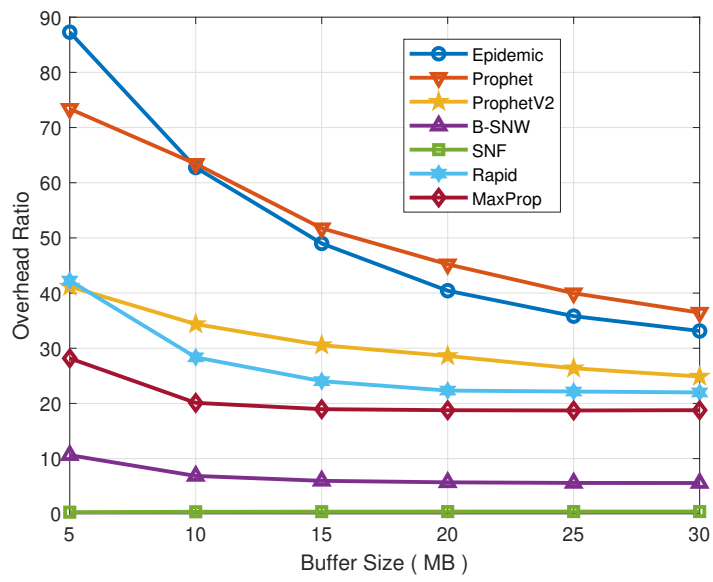

(a) Overhead ratio with changing buffer.

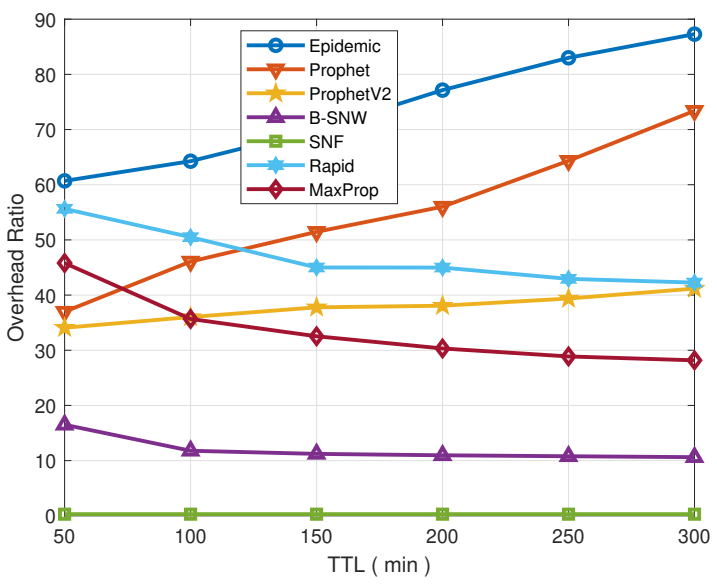

(b) Overhead ratio with varying TTL.

FIG. 5.9. Overhead with changing buffer and TTL.

social aware routing strategies.

Authors' Contribution. Md. Sharif Hossen performed the primary literature review, data collection, experiments, and also drafted the manuscript. Muhammad Sajjadur Rahim has conducted overall supervision of the research works and also suggested modifications to improve the quality of the manuscript. Both authors have read and approved the final manuscript.

Acknowledgements. This research was partially supported by the Government of the Peoples' Republic of Bangladesh. We would like to thank Bangladesh government for providing us funding on ICT-2015 research fellowship.

\section{REFERENCES}

[1] X. Chen, C. Shang, B. Wong, W. Li and S. Oh, Efficient Multicast Algorithms in Opportunistic Mobile Social Networks using Community and Social Features, Els. J. of Computer Networks, (2016), pp. 1389-1286.

[2] R. I. Ciobanu, R. C. Marin, C. Dobre, V. Cristea and C. X. Mavromoustakis, OnSiDE: Socially-aware and Interest-based Dissemination in Opportunistic Networks, IEEE Net. Operations and Management Sym. (NOMS), Krakow, Poland, (2014), pp. 1-6.

[3] J. Fan, J. Chen, Y. Du, W. Gao, J. Wu and Y. Sun, Geocommunity-Based Broadcasting for Data Dissemination in Mobile Social Networks, IEEE Tran. Parallel Distributed Syst., 24 (4), (2013), pp. 734-743.

[4] F. D. Rango F.D., A. Socievole and S. Marano, Exploiting Online and Offline Activity-Based Metrics for Opportunistic Forwarding, Springer J. of Wireless Networks, 21 (4), (2015), pp. 1163-1179.

[5] A. Socievole, E. Yoneki, F. D. Rango and J. Crowcroft, ML-SOR: Message Routing using Multi-layer Social Networks in Opportunistic Communications, Els. Jnl. of Comp. Net., 81 (22), (2015), pp. 201-219.

[6] J. Wu And Y. Wang, Opportunistic Mobile Social Networks, Taylor \& Francis CRC Press (2014).

[7] M. Xiao, J. Wu and L. Huang, Community-aware Opportunistic Routing in Mobile Social Networks, IEEE Trans. on Computers, 63 (7), (2014), pp. 1682-1695.

[8] H. Zhou, J. Chen, J. Fan, Y. Du and S. K. Das, ConSub: Incentive-based Content Subscribing in Selsh Opportunistic Mobile Networks, IEEE Journal on Selected Areas in Communication, (2013), pp. 669-679.

[9] K. Zhu, W. Li, X. Fu And L. Zhang, Data Routing Strategies in Opportunistic Mobile Social Networks: Taxonomy and Open Challenges, Computer Networks: The Int. Jnl. of Com. and Tel. Net., (2015), pp. 183-198.

[10] K. FALL, A Delay-Tolerant Network Architecture for Challenged Internets, In Proc. of ACM SIGCOMM, Germany, (2003), pp. 27-34.

[11] T. Spyropoulos, K. Psounis and C. X. Raghavendra, Single-Copy Routing in Intermittently Connected Mobile Networks, In Proc. of IEEE SAHCN, Santa Clara, CA, USA, (2005), pp. 235-244.

[12] G. E. Prescott, S. A. Smit And K. Moe, Real-time Information System Technology Challenges for NASAs Earth Science Enterprise In Proc. of IEEE RTSS, Arizona, (1999). 
[13] P. Juang, H. Oki, Y. Wang, M. Martonosi, L. S. Peh And D. Rubenstein, Energy-Efficient Computing for Wildlife Tracking: Design Tradeoffs and Early Experiences with Zebranet In Proc. of ACM ASPLOS, San Jose, California, USA, 30 (5), (2002), pp. 96-107.

[14] J. Ötт And D. Kutscher, A Disconnection-Tolerant Transport for Drivethru Internet Environments, In Proc. of IEEE INFOCOM Annual Joint Conf. of the IEEE Com. and Comm. Societies. Miami, FL, USA, (2005), pp. 1849-1862.

[15] T. Spyropoulos, K. Psounis And C. S. Raghavendra, Efficient Routing in Intermittently Connected Mobile Networks: the Single-Copy Case, IEEE/ACM Trans. on Net., 16 (1), (2008), pp. 63-76.

[16] K. Zhu, W. Li And X. Fu, SMART: A Social- and Mobile-Aware Routing Strategy for Disruption-Tolerant Networks, IEEE Trans. on Veh. Tech., 63 (7), (2014), pp. 3423-3434.

[17] M. Liu, Y. Yang And Z. QIn, A Survey of Routing Protocols and Simulations in Delay-Tolerant Networks, In Proc. of WASA, Springer Berlin, Heidelberg, vol. 6843, (2011), pp. 243-253.

[18] M. J. F. Alenazi, Y. Cheng, D. Zhang And J. P. G. Sterbenz, Epidemic Routing Protocol Implementation in ns-3, In Proc. of ACM WNS3, Barceloan, (2015), Spain, pp. 83-90.

[19] W. Moreira And P. Mendes, Social-Aware Forwarding in Opportunistic Wireless Networks: Content Awareness or Obliviousness?, In Proc. of IEEE WoWMoM, Sydney, NSW, Australia, (2014).

[20] R. I. Ciobanu , C. Dobre and V. Cristea, SPRINT: Social Prediction-Based Opportunistic Routing, In Proc. of IEEE WoWMoM, Madrid, Spain, (2013).

[21] L. Pelusi, A. Passarella and M. Conti, Opportunistic Networking: Data Forwarding in Disconnected Mobile Ad Hoc Networks, In Proc. of IEEE Comm. Magazine, 44 (11), (2006), pp. 134-141.

[22] S. Misra, B. K. Saha And S. PaL, Opportunistic Mobile Networks: Advances and Applications, Chapter 2, Springer International Publishing, Computer Communications and Networks, (2016).

[23] Z. Zhang, Routing in Intermittently Connected Mobile Ad-hoc Networks and Delay Tolerant Networks: Overview and Challenges, IEEE Com. Sur. \& Tut., vol. 8, (2006).

[24] A. Chaintreau, P. Hui, J. Crowcroft, C. Diot, R. Gass And J. Scott, Impact of Human Mobility on Opportunistic Forwarding Algorithms IEEE Tran. on Mob. Com., vol. 6, (2007).

[25] J. Miaon, O. Hasana, S. B. Mokntara, L. Bruniea and G. Gianini, A delay and cost balancing protocol for message routing in mobile delay tolerant networks, Els. J. of Ad Hoc Net., (2015), pp. 430-443.

[26] S. Jain, K. Fall And R. Patra, Routing in a Delay-Tolerant Network, In Proc. of ACM SIGCOMM, Portland, (2004), pp. $145-158$.

[27] M. Grossglauser And D. Tse D., Mobility increases the capacity of ad hoc wireless networks, IEEE/ACM Transaction on Networking, USA, (2002), pp. 477-486.

[28] A. Doria, M. Uden And D. P. Pandey, Providing Connectivity to the Saami Nomadic Community, In Proc. of ICOCDSI, Bangalore, India (2002).

[29] L. Pelusi, A. Passarella and M. Conti, Opportunistic Networking: Data Forwarding in Disconnected Mobile Ad-hoc Networks, IEEE Comm. Mag., 44 (11), (2006), pp. 134-141.

[30] D. Henriksson, T. F. Abdelzaher and R. K. Ganti, A Caching-Based Approach to Routing in Delay-Tolerant Networks In Proc. of ICCCN, Honolulu, HI, USA, (2007), pp. 69-74.

[31] A. KerÄnen, J. ÖTt And T. KÄrkKÄInen, The ONE Simulator for DTN Protocol Evaluation, In Proc. of ICSTT, Rome, Italy, (2009).

[32] A. VAhdat And D. Becker, Epidemic Routing for Partially Connected Ad-hoc Networks, Dept. of Com. Sci., Duke University, Tech. Rep., (2000).

[33] A. Lindgren, A. Doria And O. Scheln, Probabilistic Routing in Intermittently Connected Networks, ACM SIGMOBILE Mobile Compu. and Communs. Rev., 7 (3), (2003), pp. 19-20.

[34] S. Grasic, E. Davies, A. Lindgren And A. Doria, The Evolution of a DTN Routing ProtocolPRoPHETv2, ACM SiGCOM, Las Vegas, Nevada, USA, (2011), pp. 27-30.

[35] A. Balasubramanian, B. N. Levine and A. Venkataramani, DTN Routing as a Resource Allocation Problem, In Proc. of ACM SIGCOMM, Kyoto, Japan, (2007), pp. 373-384.

[36] J. Burgess, B. Gallagher, D. Jensen and B. N. Levine, MaxProp: Routing for Vehicle-Based Disruption-Tolerant Networks, In Proc. of IEEE INFOCOM, Barcelona, Spain, (2006).

[37] T. Spyropoulos, K. Psounis And C. S. Raghavendra, Spray and Wait: An Efficient Routing Scheme for Intermittently Connected Mobile Networks, In Proc. of ACM SIGCOMM WDTN, USA, (2005), pp. 252-259.

[38] T. Spyropoulos, K. Psounis and C. S. Raghavendra, Spray and Focus: Efficient Mobility-Assisted Routing for Heterogeneous and Correlated Mobility, In Proc. of IEEE PerCOMW, White Plains, NY, USA, (2007).

[39] Project page of the ONE simulator, https://www.netlab.tkk.fi/tutkimus/dtn/theone/.

[40] A. Kernen, T. KrkKinen And J. ÖTt,Simulating Mobility and DTNs with the ONE, J. of Comm., 5(2), (2010), pp. 92-105.

[41] L. Song And D. F. Kotz, Evaluating Opportunistic Routing Protocols with Large Realistic Contact Traces, In Proc. of ACM CHANTS, Montreal, Quebec, Canada, (2007), pp. 35-42.

[42] L. You, J. Li, C. Wei, C. Dai, J. Xu And L. Hu, A Hop Count Based Heuristic Routing Protocol for Mobile Delay Tolerant Network, The Scientific World Journal, (2014).

[43] H. Theus, T. Spyropoulos And F. Legendre, Putting Contacts into Context: Mobility Modeling beyond Inter-Contact Times, ACM Int. Sym. Mob. Ad Hoc Net. and Com., Paris, France, (2011), pp. 1-8.

[44] E. M. Royer, P. M. Melliar-Smith And L. E. Moser, An Analysis of the Optimum Node Density for Ad hoc Mobile Networks, IEEE ICC, Helsinki, Finland, vol. 3, (2001), pp. 857-861.

[45] Z. J. HaAs, A New Routing Protocol for the Reconfigurable Wireless Networks, In Proc. of IEEE ICUPC, San Diego, CA, USA, vol. 2, (1997), pp. 562-566. 
[46] C. Bettstetter, Mobility Modeling in Wireless Networks: Categorization, Smooth Movement, and Border Effects, In Proc. of ACM SIGMOBILE Mob. Compu. and Comms. Rev., 5 (3), (2001), pp. 55-66.

[47] A. Kernen And J. ÖTT, Increasing Reality for DTN Protocol Simulations. Technical Report, Networking Laboratory, Helsinki University of Technology, (2007)

Edited by: Khaleel Ahmad

Received: Nov 6, 2018

Accepted: Feb 20, 2019 